\title{
DEPOLARIZATION BY ELECTRICAL WAVES
}

\section{BY WILDER D. BANCROFT}

Since we know now that hydrogen over-voltage is due to the concentration of electrically neutral monatomic hydrogen at the cathode rising above the equilibrium value, ${ }^{1}$ it is possible to account for a phenomenon which appears to have puzzled people considerably, namely, the effect of electrical waves on over-voltage. Bennewitz ${ }^{2}$ electrolyzed sulphuric acid using a very small platinum anode. When this anode was exposed to strong electrical waves, the potential against a hydrogen electrode was found to be $1.28,1.27,1.24$ volts. Since the theoretical value for the hydrogen-oxygen gas cell is 1.23 volts, this means that the over-voltage at the oxygen electrode has been reduced to the negligible value of $0.01-0.05$ volt. Bennewitz offered no explanation for the phenomenon and did not even discuss whether it was general or specific.

I think that we are safe in assuming that the phenomenon is general and not limited to this special case. It is, therefore, necessary to find a general explanation. From experiments on the disruptive discharge in gases, Schuster ${ }^{3}$ concluded that there must be a layer of adsorbed gas on the electrodes, having a high inductive capacity and, therefore, offering an increased resistance to the discharge. If this is true, it follows from the theorem of Le Chatelier that an electrical stress will tend to remove the film of adsorbed gas. ${ }^{4}$ This conclusion can be used to account for the behavior of fountains, impinging jets, rolling drops, and soap bubbles, when electrified slightly. It is, therefore, a good working hypothesis. If an electrical stress tends to remove a gas from the surface of a solid, electrical waves will tend to remove active oxygen from the surface

${ }^{1}$ Bennett: Jour. Phys. Chem., 20, 296 (I9I6).

2 Zeit. phys. Chem., 72, 223 (I IIO); cf. Foerster: "Elektrochemie wässeriger Lösungen," 293 (1915).

${ }^{3}$ Phil. Mag., (5) 29, 197 (I890).

${ }^{4} \mathrm{Cf}$. Bancroft: Jour. Phys. Chem., 20, 18 (1916). 
of an electrode and will, therefore, cut down the over-voltage, which is what Bennewitz found. We ought to find also that electrical waves cut down the hydrogen over-voltage. This has been shown to be the case by Rothmund. ${ }^{1}$ whose paper seems to have been overlooked both by Bennewitz and by Foerster. More recently Archibald and von Wartenberg? have found that the anodic over-voltage is cut down when an alternating current is superposed on a direct current. Reitlinger ${ }^{3}$ continued the work of von Wartenberg and sums up his results as follows: "Alternating current destroys the over-voltage due to direct current electrolysis, the potential difference between the electrode and the solution being decreased. The lower potential makes possible the isolation of intermediate products which are destroyed at higher potentials. When an alternating current is superposed on a direct current, there is an increase in the yield of the intermediate products and a decrease in the amount of the final products. Thus ozone is formed instead of persulphuric acid when sulphuric acid is electrolyzed. When alcohols are electrolyzed, aldehydes are formed instead of the corresponding acids. In an indirect way this confirms the experiments of Dony-Hénault, for he showed that aldehyde is formed from alcohol when the potential is kept sufficiently low, whereas acetic acid is the chief product when the potential is higher.

"It is only the negative portion of the alternating current which causes the lowering of the potential and makes possible the formation of intermediate products in relatively large amount. This is made clear in the experiments on ozone, for the potential falls and the yield of ozone increases only when the anode becomes cathode intermittently. With highfrequency currents the depolarizing action is less, owing to the great displacement of phase caused by the capacity of the electrodes."

\footnotetext{
1 Drude's Ann., I5, 193 (1904).

2 Zeit. Elektrochemie, I7, 812 (I9II).

3 Ibid., 20, 26I (I914).
} 
About a year ago, Ghosh ${ }^{1}$ published a paper on the effect of superposing an alternating current from an induction coil on a direct current. He found that the cathode effect is slight with a platinum black cathode where the over-voltage is negligible, and is large with a mercury cathode where the overvoltage is high. He showed that the effect was due to the cutting down of the polarization; but he was unable to account for the phenomenon. "The alternating current acts primarily on the surfaces of the electrodes. Of course, it is very difficult to suggest how it facilitates the liberation of the ions at the electrode surfaces, since we have not got very definite views as to the cause of the over-voltage phenomenon. It is not easy to imagine how an alternating current can destroy the supersaturation on the electrode surface, which is generally ascribed to be the cause of the over-voltage. The hypothesis that the alternating current somehow alters the nature of the electrode surface appears to be most reasonable."

As a matter of fact, the one assumption made by Schuster accounts for all these facts as well as for many others. An interesting corollary from the work of Rothmund, Bennewitz, Archibald and von Wartenberg, Reitlinger, and Ghosh is that with a sufficient depolarizing action at the anode lead peroxide should not be formed with lead electrodes in sulphuric acid because the formation of lead peroxide calls for an over-voltage of about half a volt." This seems to have been confirmed experimentally by Ruer, ${ }^{3}$ who used a very dilute sulphuric acid ( 2.5 percent) however, and who seems to have obtained some lead peroxide at times. These experiments should be repeated with a more concentrated acid. With alternating current alone, lead sulphate is formed and no lead peroxide. ${ }^{4}$

Sufficiently strong electrical waves should also prevent the formation of sodium amalgam at a mercury cathode.

'Jour. Am. Chem. Soc., 37, 733 (1915).

2 Dolezalek: Zeit. Elektrochemie, 5, 537 (I 899).

${ }^{3}$ Zeit. phys. Chem., 44, 105 (1903).

${ }^{4}$ Cf. Schluederberg: Jour. Phys. Chem., I2, 623 (Igo8). 
Zinc, cadmium, and nickel should dissolve readily in sulphuric acid under similar conditions. Since lead peroxide is theoretically instable, there is a possibility of causing it to break down into lead oxide and oxygen. This seems not to have happened in Reitlinger's work for he used lead peroxide anodes in some experiments. Until we can make this reaction occur, it is hardly worth while to speculate on the fascinating possibility of making potassium chlorate break up at ordinary temperatures into potassium chloride and oxygen, to say nothing of the more remote possibility of eliminating passive resistances to change in the case of organic compounds.

The experiments of Margules ${ }^{1}$ and Ruer ${ }^{2}$ on the dissolving of platinum find their explanation in the cutting down of the over-voltage, as was recognized explicitly by Reitlinger. ${ }^{3}$ With a direct current we get oxidation to a higher and insoluble stage. With alternating current the over-voltage is decreased and we get little or none of the insoluble compound.

There is one other point of distinct interest with respect to electric waves. The more recent determinations of overvoltage have given lower values than those obtained by Caspari. ${ }^{4}$ This may be due to experimental error on Caspari's part. On the other hand many of the recent determinations have been made with an intermittent direct current, the polarized electrode being connected with the potentiometer circuit during the breaks. It is possible that the polarization might have been cut down somewhat by induced currents during the make and break. If this were so, the measurements by this method would be in error. It may be that the error from this source is negligible; but this should be proved and not assumed.

The general results of this paper are that electrical waves must cause depolarization if we admit that electrical stress

${ }^{1}$ Weid. Ann., 65, 629;66, 540 (1898).

2 Zeit. phys. Chem., 44, 8I; Zeit. Elektrochemie, 9, 235 (1903); II, IO, 66I (1905); Haber: Zeit. anorg. Chem., 5I, 365 (1906).

${ }^{3}$ Zeit. Elektrochemie, 20, 261 (1914).

${ }^{4}$ Cf. Bennett: Jour. Phys. Chem., 20, 296 (1916). 
cuts down the adsorption of a gas by a solid. This assumption is a necessary consequence of Schuster's work on disruptive discharges and has been shown to account for the behavior of fountains, impinging jets, rolling drops, and soapbubbles, when electrified slightly. It is shown that electrical waves do decrease over-voltage at the cathode and at the anode, and that the assumption accounts for all the facts so far known. It is also pointed out that measurements of decomposition voltage involving the use of an intermittent direct current are subject to an error which is perhaps not negligible.

Cornell Unicersity 